\title{
An efficient finite difference scheme for the 2D sine-Gordon equation
}

\author{
Xiaorong Kang ${ }^{\mathrm{a}}$, Wenqiang Feng ${ }^{\mathrm{b}}$, Kelong Cheng ${ }^{\mathrm{a}}$, Chunxiang Guo ${ }^{\mathrm{c}, *}$ \\ a School of Science, Southwest University of Science and Technology, Mianyang, Sichuan 621010, China. \\ ${ }^{b}$ Department of Mathematics, University of Tennessee, Knoxville, Tennessee 37996, USA. \\ ${ }^{c}$ School of Business, Sichuan University, Chengdu, Sichuan 610064, China.
}

Communicated by X.-J. Yang

\begin{abstract}
We present an efficient second-order finite difference scheme for solving the 2D sine-Gordon equation, which can inherit the discrete energy conservation for the undamped model theoretically. Due to the semi-implicit treatment for the nonlinear term, it leads to a sequence of nonlinear coupled equations. We use a linear iteration algorithm, which can solve them efficiently, and the contraction mapping property is also proven. Based on truncation errors of the numerical scheme, the convergence analysis in the discrete $l^{2}$-norm is investigated in detail. Moreover, we carry out various numerical simulations, such as verifications of the second order accuracy, tests of energy conservation and circular ring solitons, to demonstrate the efficiency and the robustness of the proposed scheme. (C)2017 All rights reserved.
\end{abstract}

Keywords: 2D sine-Gordon equation, conservative, difference scheme, linear iteration, convergence.

2010 MSC: 65M06, 65M12.

\section{Introduction}

In this paper, we consider the following 2D sine-Gordon equation,

$$
u_{t t}+\beta u_{t}-\alpha \Delta u=-\phi(x, y) \sin u+F(x, y, t), \quad(x, y) \in \Omega, \quad t \geqslant 0,
$$

with initial conditions

$$
u(x, y, 0)=\varphi_{1}(x, y), \quad u_{t}(x, y, 0)=\varphi_{2}(x, y), \quad(x, y) \in \Omega,
$$

and the boundary condition

$$
\left.u\right|_{\partial \Omega}=G(t), \quad t \geqslant 0,
$$

where $\Omega=[0, \mathrm{~L}]^{2}$. This equation has attracted much attention due to the presence of soliton solutions and has a great deal of applications in the propagation of fluxons in Josephson junctions between two

\footnotetext{
${ }^{*}$ Corresponding author

Email addresses: kangxiaorong@swust.edu.cn (Xiaorong Kang), wfeng1@vols.utk.edu (Wenqiang Feng), zhengkelong@swust.edu.cn (Kelong Cheng), guocx70@163.com (Chunxiang Guo)

doi:10.22436/jnsa.010.06.14
} 
superconductors [16], the motion of a rigid pendulum attached to a stretched wire [20], dislocations in crystals and the stability of fluid motions. Nowadays, it has become one of paradigms of the nonlinear dynamical system to describe many different physical phenomena [21]. In (1.1), $\phi(x, y)$ is a nonnegative function with finite bound $\phi_{0}$ and may be interpreted as the Josephson current density, while $\varphi_{1}(x, y)$ and $\varphi_{2}(x, y)$ represent wave modes or kinks and velocity, respectively. In particular, when $\beta=0,(1.1)$ reduces to the undamped sine-Gordon equation,

$$
u_{t t}-\alpha \Delta u=-\phi(x, y) \sin u+F(x, y, t) .
$$

If $F=0$ and $G$ is periodic or homogeneous, one of the main properties of the undamped sine-Gordon equation (1.4) has the conservation for the energy defined as follows [3, 4],

$$
\begin{aligned}
E(t) & =\frac{1}{2} \int_{\Omega}\left[\left|u_{t}\right|^{2}+|\nabla u|^{2}+2 \phi(1-\cos u)\right] d x d y \\
& =\frac{1}{2}\left(\left\|u_{t}\right\|_{L^{2}(\Omega)}^{2}+\|\nabla u\|_{L^{2}(\Omega)}^{2}\right)+\int_{\Omega} \phi(1-\cos u) d x d y,
\end{aligned}
$$

which is not valid for the damped system (1.1).

Recently, various analytical and numerical methods have been proposed for the numerical solution of partial differential equations, for example, the integral transform [22-24] and traveling-wave technologies $[25,26]$. Analytical solutions to the unperturbed sine-Gordon equation with zero damping have been obtained by Lambs methods [27] and Bäcklund transformations. Many efforts have been attempted to develop numerical methods, such as the finite difference method [3, 4, 9], the time-splitting pseudospectral and spectral method [2], the finite element method [1], the mesh-free reproducing kernel particle Ritz method [7], the local weak meshless method [10], the boundary element method [11], the differential quadrature method [14] and the radial basis functions method [12] for the 2D sine-Gordon equation. However, there exist few available error estimate results in the above-mentioned works for the 2D case. The main reason is that the techniques used for 1D case can not be extended trivially to high dimensions because of the difficulty in obtaining the a priori uniform estimate of the numerical solution.

Since the undamped sine-Gordon equation is a conservative system, it should be pointed out that a conservative numerical scheme performs better than a nonconservative one. The key is that it can preserve some invariant properties of the differential equation and capture physical procedures with more details [15]. Moreover, there has been growing interest in conservative numerical methods for solving partial differential equations. For example, Klein-Gordon equation [17], the high frequency wave phenomena $[6,13,18,28]$, the phase field crystal model [19], and so forth. As for the sine-Gordon equation, to our knowledge, only a few results considered this vital property. Although many verification results of the discrete energy for various numerical methods are reported [2-4], the analysis at a theoretical level was hardly shown.

The main purpose of this paper is to present a second-order semi-implicit finite difference scheme for numerical solutions of the 2D sine-Gordon equation (1.1)-(1.3). There are three main features to this work. The first is that the proposed scheme can admit the discrete energy conversation for the undamped case (1.4) at a theoretical level, which has not yet been reported in the existing literatures. The second feature of this work is the linear iteration algorithm introduced [18] to solve efficiently the nonlinear system at each time step due to the implicit treatment of the nonlinear term. Meanwhile, a careful analysis shows a contraction mapping property of this iteration under the given constrain for the time step. Finally, we provided a detailed convergence analysis for the second-order scheme in the $l^{2}$-norm.

The remainder of the paper is organized as follows. In Section 2, the second-order finite difference scheme is proposed and the energy conservation property for the undamped system is proven. The linear iteration algorithm and the corresponding theoretical analysis of the contraction mapping are given in Section 3. Truncation errors and the convergence analysis are discussed in Section 4. Some numerical simulation results are given to demonstrate the efficiency of the linear iteration solver and the convergence of the scheme in Section 5. Finally, some conclusions are made in Section 6. 


\section{Numerical scheme and energy conservation}

\subsection{Second order finite difference scheme}

Let $v=u_{t}$. (1.1) can be rewritten as,

$$
\begin{aligned}
v_{t}+\beta u_{t}-\alpha \Delta u & =-\phi(x, y) \sin u+F(x, y, t), \\
v & =u_{t} .
\end{aligned}
$$

Then, for the given 2D domain $\Omega$, define the uniform numerical grid $\left(x_{i}, y_{j}\right)$ with $\Delta x=\Delta y=h$ for simplicity of presentation. Let $M_{x}=M_{y}=M$ and $M \cdot h=L$ such that $x_{i}=i h, i=0,1, \cdots, M, y_{j}=j h, j=$ $0,1, \cdots, M$. For a fixed time $T$, let $\Delta t$ be the step size for temporal direction, $t^{n}=n \Delta t, n=0,1,2, \cdots, N$, $N=\left[\frac{T}{\Delta t}\right], u_{i, j}^{n} \approx u\left(x_{i}, y_{j}, t^{n}\right)$. Denote $\Delta_{h}=D_{x x}+D_{y y}$ as the standard second order difference operator with

$$
\begin{aligned}
D_{x} u & =\frac{u_{i+1, j}-u_{i, j}}{h}, & D_{y} u & =\frac{u_{i, j+1}-u_{i, j}}{h}, \\
D_{x x} u & =\frac{u_{i+1, j}-2 u_{i, j}+u_{i-1, j}}{h^{2}}, & D_{y y} u & =\frac{u_{i, j+1}-2 u_{i, j}+u_{i, j-1}}{h^{2}} .
\end{aligned}
$$

The second order finite difference scheme is presented at a point-wise level as follows,

$$
\begin{aligned}
\frac{v^{n+1}-v^{n}}{\Delta t}+\beta \frac{u^{n+1}-u^{n}}{\Delta t}-\frac{\alpha}{2} \Delta_{h}\left(u^{n+1}+u^{n}\right) & =\phi \frac{\cos \left(u^{n+1}\right)-\cos \left(u^{n}\right)}{u^{n+1}-u^{n}}+F^{n+\frac{1}{2}}, \\
\frac{u^{n+1}-u^{n}}{\Delta t} & =\frac{v^{n+1}+v^{n}}{2},
\end{aligned}
$$

with discrete initial conditions

$$
u_{i, j}^{0}=\varphi_{1}\left(x_{i}, y_{j}\right), \quad v_{i, j}^{0}=\varphi_{2}\left(x_{i}, y_{j}\right),
$$

and the boundary condition

$$
\left.u_{i, j}^{n}\right|_{\partial \Omega}=G\left(x_{i}, y_{j}, t^{n}\right), \quad\left(x_{i}, y_{j}\right) \in \partial \Omega,
$$

where $F^{n+\frac{1}{2}}=F\left(x_{i}, y_{j}, t^{n+\frac{1}{2}}\right)$. Obviously, (2.4) can be reformulated as

$$
v^{n+1}=\frac{2\left(u^{n+1}-u^{n}\right)}{\Delta t}-v^{n} .
$$

Substituting (2.5) into (2.3) yields that

$$
\frac{2 u^{n+1}}{\Delta t^{2}}+\frac{\beta}{\Delta t} u^{n+1}-\frac{\alpha}{2} \Delta_{h} u^{n+1}=\phi \frac{\cos \left(u^{n+1}\right)-\cos \left(u^{n}\right)}{u^{n+1}-u^{n}}+\frac{\beta}{\Delta t} u^{n}+\frac{\alpha}{2} \Delta_{h} u^{n}+k\left(u^{n}, v^{n}\right)+F^{n+\frac{1}{2}},
$$

where $\kappa\left(u^{n}, v^{\mathfrak{n}}\right)=\frac{\frac{2 u^{n}}{\Delta t}+2 v^{n}}{\Delta t}$. (2.6) is nonlinear and can be solved implicitly by a linear iteration algorithm introduced in the next section. Following $\mathfrak{u}^{n+1}$ is solved, $v^{n+1}$ can be computed explicitly by (2.5).

Remark 2.1. The main idea to deal with the sine nonlinearity was first introduced by Strauss and Vázquez [17] to compute numerical solutions of a nonlinear Klein-Gordon equation in which a polynomial nonlinear term is involved. In fact, this subtle technique can achieve perfect numerical solutions for the conservative model and has been extensively studied for some nonlinear problems, such as the CahnHilliard type equation $[5,8]$.

\subsection{Discrete energy conservation for the undamped equation}

As above mentioned, the undamped sine-Gordon equation with certain boundary conditions admits the property of energy conservation. Here, as the special case of the numerical scheme (2.3) and (2.4), the difference scheme for the undamped equation is conservative for the discrete energy. 
Letting $F(x, y, t)=0$ and $G(t)=0$, from (1.4), we have

$$
u_{t t}-\alpha \Delta u=-\phi(x, y) \sin u,
$$

which satisfies the conservative law (1.5). And also, the corresponding numerical finite difference scheme is simplified as follows,

$$
\begin{aligned}
\frac{v^{n+1}-v^{n}}{\Delta t}-\frac{\alpha}{2} \Delta_{h}\left(u^{n+1}+u^{n}\right) & =\phi \frac{\cos \left(u^{n+1}\right)-\cos \left(u^{n}\right)}{u^{n+1}-u^{n}}, \\
\frac{u^{n+1}-u^{n}}{\Delta t} & =\frac{v^{n+1}+v^{n}}{2} .
\end{aligned}
$$

Next, we introduce the $l^{2}$-norm and the $l^{2}$ inner product. For any two homogeneous (or periodic) grid functions $f$ and $g$, define the discrete $l^{2}$ inner product and the discrete $l^{2}$-norm, respectively, as

$$
\langle f, g\rangle=h^{2} \sum_{i, j=0}^{M} f_{i, j} g_{i, j}, \quad\|f\|_{2}=\sqrt{\langle f, f\rangle}
$$

and the following summation by parts is also straightforward,

$$
\left\langle\Delta_{h} f, g\right\rangle=-\left\langle\nabla_{h} f, \nabla_{h} g\right\rangle,
$$

with

$$
\begin{aligned}
& \left\|\nabla_{h} f\right\|_{2}^{2}=\left\|D_{x} f\right\|_{2}^{2}+\left\|D_{y} f\right\|_{2}^{2} \\
& \left\|D_{x} f\right\|_{2}^{2}=h^{2} \sum_{i, j=0}^{M}\left(f_{i+1, j}-f_{i, j}\right)^{2} / h^{2}, \quad\left\|D_{y} f\right\|_{2}^{2}=h^{2} \sum_{i, j=0}^{M}\left(f_{i, j+1}-f_{i, j}\right)^{2} / h^{2} .
\end{aligned}
$$

Theorem 2.2. The scheme (2.7)-(2.8) is conservative for the discrete energy, namely,

$$
\mathcal{E}^{n}=\frac{1}{2}\left\|v^{n}\right\|_{2}^{2}+\frac{\alpha}{2}\left\|\nabla_{h} u^{n}\right\|_{2}^{2}+h^{2} \sum_{i, j=0}^{M}\left(\phi\left(1-\cos \left(u^{n}\right)\right)\right)_{i j}=\mathcal{E}^{0}
$$

for $\mathrm{n}=1,2, \cdots, \mathrm{N}$.

Remark 2.3. The main purpose of taking this discrete energy form is to be in accordance with its continuous definition (1.3). Alternatively, if we delete the constant in the term $\phi\left(1-\cos \left(u^{n}\right)\right)$, the new discrete energy is also conservative.

Remark 2.4. In [2], the authors have simulated the discrete energy which looks to likely be conservative. However, it might be caused by the high accuracy of the proposed pseudospectral method. As we know, the explicit numerical scheme could not ensure the conversation generally.

Proof. Taking the inner product of (2.7) with $u^{n+1}-u^{n}$ yields

$$
\begin{aligned}
& \left\langle\frac{v^{n+1}-v^{n}}{\Delta t}, u^{n+1}-u^{n}\right\rangle-\frac{\alpha}{2}\left\langle\Delta_{h}\left(u^{n+1}+u^{n}\right), u^{n+1}-u^{n}\right\rangle \\
& -\left\langle\phi \frac{\cos \left(u^{n+1}\right)-\cos \left(u^{n}\right)}{u^{n+1}-u^{n}}, u^{n+1}-u^{n}\right\rangle=0 .
\end{aligned}
$$

For the first term, we have

$$
\begin{aligned}
\left\langle\frac{v^{n+1}-v^{n}}{\Delta t}, u^{n+1}-u^{n}\right\rangle=\left\langle v^{n+1}-v^{n}, \frac{u^{n+1}-u^{n}}{\Delta t}\right\rangle & =\frac{1}{2}\left\langle v^{n+1}-v^{n}, v^{n+1}+v^{n}\right\rangle \\
& =\frac{1}{2}\left(\left\|v^{n+1}\right\|_{2}^{2}-\left\|v^{n}\right\|_{2}^{2}\right),
\end{aligned}
$$


where (2.8) is applied in the second step. According to (2.7), the second term can be analyzed as

$$
-\frac{\alpha}{2}\left\langle\Delta_{h}\left(u^{n+1}+u^{n}\right), u^{n+1}-u^{n}\right\rangle=\frac{\alpha}{2}\left(\left\|\nabla_{h} u^{n+1}\right\|_{2}^{2}-\left\|\nabla_{h} u^{n}\right\|_{2}^{2}\right) .
$$

Moreover, for the nonlinear term, we obtain the following result,

$$
-\left\langle\phi \frac{\cos \left(u^{n+1}\right)-\cos \left(u^{n}\right)}{u^{n+1}-u^{n}}, u^{n+1}-u^{n}\right\rangle=h^{2} \sum_{i, j=0}^{n} \phi_{i, j}\left(\left(1-\cos u^{n+1}\right)-\left(1-\cos u^{n}\right)\right)_{i, j} .
$$

By the definition of $\mathcal{E}^{n},(2.9)$ is obtained from (2.10)-(2.13).

\section{Linear iteration algorithm}

Since the nonlinear term $\cos \left(\mathrm{u}^{\mathrm{n}+1}\right)$ is treated implicitly in (2.6), it leads to a sequence of nonlinear coupled equations. In order to solve the nonlinear system (2.6) arising from the implicit treatment, we propose the following linear iteration algorithm:

$$
\begin{aligned}
& \frac{2 u^{n+1,(m+1)}}{\Delta t^{2}}+\frac{\beta}{\Delta t} u^{n+1,(m+1)}-\frac{\alpha}{2} \Delta_{h} u^{n+1,(m+1)} \\
& =\phi \frac{\cos \left(u^{n+1,(m)}\right)-\cos \left(u^{n}\right)}{u^{n+1,(m)}-u^{n}}+\frac{\beta}{\Delta t} u^{n}+\frac{\alpha}{2} \Delta_{h} u^{n}+k\left(u^{n}, v^{n}\right)+F^{n+\frac{1}{2}},
\end{aligned}
$$

where $u^{n+1,(m)}$ denotes the approximation solution at the $m$-th iteration.

Theorem 3.1. The linear iteration scheme (3.1) is a contraction mapping, provided that $\Delta \mathrm{t}<\frac{\beta+\sqrt{\beta^{2}+8 \phi_{0}}}{2 \phi_{0}}$.

Proof. Define the iteration error of each stage via

$$
e^{(m)}=u^{n+1,(m)}-u^{n+1}
$$

where $u^{n+1,(m)}$ is the $m$-th iteration result generated by the linear iteration scheme (3.1). Subtracting (3.1) from (2.6) leads to

$$
\left(\frac{2}{\Delta t^{2}}+\frac{\beta}{\Delta t}-\frac{\alpha}{2} \Delta_{h}\right) e^{(m+1)}=\phi\left(\frac{\cos \left(u^{n+1,(m)}\right)-\cos \left(u^{n}\right)}{u^{n+1,(m)}-u^{n}}-\frac{\cos \left(u^{n+1}\right)-\cos \left(u^{n}\right)}{u^{n+1}-u^{n}}\right) .
$$

Taking the inner product of (3.2) with $e^{(m+1)}$, we have

$$
\begin{aligned}
& \left\langle\left(\frac{2}{\Delta \mathrm{t}^{2}}+\frac{\beta}{\Delta \mathrm{t}}-\frac{\alpha}{2} \Delta_{h}\right) e^{(\mathrm{m}+1)}, \mathrm{e}^{(\mathrm{m}+1)}\right\rangle \\
& \quad=\left(\frac{2}{\Delta \mathrm{t}^{2}}+\frac{\beta}{\Delta \mathrm{t}}\right)\left\|e^{(\mathrm{m}+1)}\right\|_{2}^{2}+\frac{\alpha}{2}\left\|\nabla_{h} e^{(m+1)}\right\|_{2}^{2} \\
& \quad=\left\langle\phi\left(\frac{\cos \left(\mathrm{u}^{\mathrm{n}+1,(m)}\right)-\cos \left(\mathrm{u}^{\mathrm{n}}\right)}{\mathrm{u}^{\mathrm{n}+1,(m)}-\mathrm{u}^{\mathrm{n}}}-\frac{\cos \left(\mathrm{u}^{\mathrm{n}+1}\right)-\cos \left(\mathrm{u}^{\mathrm{n}}\right)}{\mathrm{u}^{\mathrm{n}+1}-\mathrm{u}^{\mathrm{n}}}\right), e^{(m+1)}\right\rangle .
\end{aligned}
$$

Now, we analyze the right-hand side of (3.3) in detail. For convenience, let

$$
h(x)=\frac{\cos x-\cos a}{x-a} .
$$

Using the Lagrange theorem, we obtain $h(x)=-\sin \xi$, where $\xi$ is between $x$ and $a$. We also compute the derivative of $h(x)$,

$$
h^{\prime}(x)=\frac{-(x-a) \sin x-(\cos x-\cos a)}{(x-a)^{2}}=\frac{-\sin x-\frac{(\cos x-\cos a)}{x-a}}{x-a}=\frac{-\sin x+\sin \xi}{x-a} .
$$


Applying the Lagrange theorem again for $(-\sin x+\sin \xi)$ yields

$$
\left|h^{\prime}(x)\right|=\left|\cos \xi_{1}\right| \cdot \frac{|x-\xi|}{|x-a|}<1,
$$

where $\xi_{1}$ is between $x$ and $\xi$, and the fact that $|x-\xi|<|x-a|$ is used.

Going back to (3.3) and setting $a=u^{n}$, we have

$$
\begin{aligned}
\left|\frac{\cos \left(u^{n+1,(m)}\right)-\cos \left(u^{n}\right)}{u^{n+1,(m)}-u^{n}}-\frac{\cos \left(u^{n+1}\right)-\cos \left(u^{n}\right)}{u^{n+1}-u^{n}}\right| & =\left|h\left(u^{n+1,(m)}\right)-h\left(u^{n+1}\right)\right| \\
& =\left|h^{\prime}\left(\xi_{2}\right)\right| \cdot\left|u^{n+1,(m)}-u^{n+1}\right|<\left|e^{(m)}\right|,
\end{aligned}
$$

with $\xi_{2}$ between $u^{n+1,(m)}$ and $u^{n+1}$. In turn, one can get

$$
\begin{aligned}
\left\langle\phi\left(\frac{\cos \left(u^{n+1,(m)}\right)-\cos \left(u^{n}\right)}{u^{n+1,(m)}-u^{n}}-\frac{\cos \left(u^{n+1}\right)-\cos \left(u^{n}\right)}{u^{n+1}-u^{n}}\right), e^{(m+1)}\right\rangle & \leqslant \phi_{0}\left|\left\langle e^{(m)}, e^{(m+1)}\right\rangle\right| \\
& \leqslant \frac{\phi_{0}}{2}\left(\left\|e^{(m+1)}\right\|_{2}^{2}+\left\|e^{(m)}\right\|_{2}^{2}\right),
\end{aligned}
$$

in which $\phi_{0}$ is the upper bound of $\phi$.

As a result, it follows from the combination of (3.3) and (3.5) that

$$
\left(\frac{2}{\Delta \mathrm{t}^{2}}+\frac{\beta}{\Delta \mathrm{t}}-\frac{\phi_{0}}{2}\right)\left\|e^{(\mathrm{m}+1)}\right\|_{2}^{2}+\frac{1}{2}\left\|\nabla_{\mathrm{h}} e^{(\mathrm{m}+1)}\right\|_{2}^{2} \leqslant \frac{\phi_{0}}{2}\left\|e^{(\mathrm{m})}\right\|_{2}^{2} .
$$

Therefore, the contraction mapping property can be assured if

$$
\left(\frac{2}{\Delta \mathrm{t}^{2}}+\frac{\beta}{\Delta \mathrm{t}}-\frac{\phi_{0}}{2}\right)>\frac{\phi_{0}}{2},
$$

which shows that the result is proven.

Remark 3.2. For a high dimensional problem, either the ADI scheme or the predictor-corrector scheme is often used to implement the implicit finite difference scheme. Both schemes belong to two-step method or multi-step one for reducing the dimension complexity. For 2D sine-Gordon equation, see [3, 4, 9]. Without any decompositions for 2D sine-Gordon equation, the implicit scheme can be solved efficiently by the linear iteration. Certainly, this iteration method is also applied in many numerical methods, but the contraction condition for iterations is seldom investigated. On the other hand, we also can present the high-order finite difference scheme if more complicated operators are involved, and can solve it by the iteration method.

\section{Truncation errors and the convergence analysis}

\subsection{Truncation errors}

Let $u_{e}$ and $v_{e}=\partial_{t} u_{e}$ be exact solutions of the problem (1.1)-(1.3), then truncation errors of the scheme (2.1) are obtained at discrete grid points as follows,

$$
\begin{aligned}
\frac{v_{e}^{k+1}-v_{e}^{k}}{\Delta t}+\frac{\beta}{\Delta t}\left(u_{e}^{k+1}-u_{e}^{k}\right)-\frac{\alpha}{2} \Delta_{h}\left(u_{e}^{k+1}+u_{e}^{k}\right)-\phi \frac{\cos \left(u_{e}^{k+1}\right)-\cos \left(u_{e}^{k}\right)}{u_{e}^{k+1}-u_{e}^{k}}-F^{k+\frac{1}{2}} & =\rho^{k}, \\
\frac{u_{e}^{k+1}-u_{e}^{k}}{\Delta t}-\frac{v_{e}^{k+1}+v_{e}^{k}}{2} & =s^{k} .
\end{aligned}
$$

In fact, for a given function $f(x) \in C^{5}$, one can get

$$
\frac{f(\xi)-f(\eta)}{\xi-\eta}=f^{\prime}\left(\frac{\xi+\eta}{2}\right)+\frac{1}{24} f^{\prime \prime}\left(\frac{\xi+\eta}{2}\right)(\xi-\eta)^{2}+O\left((\xi-\eta)^{4}\right) .
$$


Taking $f(x)=\cos (x), \xi=u_{e}^{k+1}$, and $\eta=u_{e}^{k}$, we have

$$
\frac{\cos \left(u_{e}^{k+1}\right)-\cos \left(u_{e}^{k}\right)}{u_{e}^{k+1}-u_{e}^{k}}=-\sin \left(\frac{u_{e}^{k+1}+u_{e}^{k}}{2}\right)+O\left(\Delta t^{2}\right)=-\sin \left(u_{e}^{k+\frac{1}{2}}\right)+O\left(\Delta t^{2}\right) .
$$

Similarly, other terms can be analyzed and the details are omitted. Hence, we obtain the following lemma.

Lemma 4.1. Suppose $\mathrm{u}_{\mathrm{e}}$ and $v_{\mathrm{e}}$ are smooth enough, then $\left|\rho_{i j}^{k}\right|+\left|s_{i j}^{k}\right|=\mathrm{O}\left(\Delta \mathrm{t}^{2}+\mathrm{h}^{2}\right)$ holds as $\Delta \mathrm{t}, \mathrm{h} \rightarrow 0$.

\subsection{Convergence analysis}

Define discrete error functions as follows,

$$
\tilde{u}^{k}=u_{e}^{k}-u^{k}, \quad \tilde{v}^{k}=v_{e}^{k}-v^{k} .
$$

Now, we present the following convergence result.

Theorem 4.2. Assume that $u_{e}$ and $v_{e}=\partial_{t} u_{e}$ are the exact solutions of the problem (1.1)-(1.3), and denote $(u, v)$ as the numerical solution given by the finite difference scheme (2.3) and (2.4). Then, we have

$$
\left\|\tilde{v}^{\mathfrak{n}}\right\|_{2}^{2}+\left\|\tilde{u}^{\mathrm{n}}\right\|_{2}^{2}+\alpha\left\|\nabla_{\mathrm{h}} \tilde{\mathrm{u}}^{\mathrm{n}}\right\|_{2}^{2} \leqslant \tilde{\mathrm{C}} \cdot \mathrm{O}\left(\Delta \mathrm{t}^{2}+\mathrm{h}^{2}\right),
$$

where the constant $\tilde{\mathrm{C}}$ is dependent on the final time $\mathrm{T}$ and is independent on $\Delta \mathrm{t}$ and $\mathrm{h}$.

Proof. Subtracting (2.1)-(2.2) from (4.1)-(4.2), respectively, we get

$$
\begin{aligned}
\frac{\tilde{v}^{k+1}-\tilde{v}^{k}}{\Delta t}+\frac{\beta}{\Delta t}\left(\tilde{u}^{k+1}-\tilde{u}^{k}\right) & -\frac{\alpha}{2} \Delta_{h}\left(\tilde{u}^{k+1}+\tilde{u}^{k}\right) \\
& -\phi\left(\frac{\cos \left(u_{e}^{k+1}\right)-\cos \left(u_{e}^{k}\right)}{u_{e}^{k+1}-u_{e}^{k}}-\frac{\cos \left(u^{k+1}\right)-\cos \left(u^{k}\right)}{u^{k+1}-u^{k}}\right)=\rho^{k}, \\
& \frac{\tilde{u}^{k+1}-\tilde{u}^{k}}{\Delta t}-\frac{\tilde{v}^{k+1}+\tilde{v}^{k}}{2}=s^{k} .
\end{aligned}
$$

Taking the inner product of (4.3) with $\tilde{u}^{k+1}-\tilde{u}^{k}$ yields

$$
\begin{aligned}
& \frac{1}{\Delta t}\left\langle\tilde{v}^{k+1}-\tilde{v}^{k}, \tilde{u}^{k+1}-\tilde{u}^{k}\right\rangle+\frac{\beta}{\Delta t}\left\langle\tilde{u}^{k+1}-\tilde{u}^{k}, \tilde{u}^{k+1}-\tilde{u}^{k}\right\rangle-\frac{\alpha}{2}\left\langle\Delta_{h}\left(\tilde{u}^{k+1}+\tilde{u}^{k}\right), \tilde{u}^{k+1}-\tilde{u}^{k}\right\rangle \\
& =\left\langle\phi\left(\frac{\cos \left(u_{e}^{k+1}\right)-\cos \left(u_{e}^{k}\right)}{u_{e}^{k+1}-u_{e}^{k}}-\frac{\cos \left(u^{k+1}\right)-\cos \left(u^{k}\right)}{u^{k+1}-u^{k}}\right), \tilde{u}^{k+1}-\tilde{u}^{k}\right\rangle+\left\langle\rho^{k}, \tilde{u}^{k+1}-\tilde{u}^{k}\right\rangle .
\end{aligned}
$$

Next, we begin to analyze the nonlinear term on the right-hand of (4.5). Noting that

$$
\begin{aligned}
& \left|\left(\frac{\cos \left(u_{e}^{k+1}\right)-\cos \left(u_{e}^{k}\right)}{u_{e}^{k+1}-u_{e}^{k}}-\frac{\cos \left(u^{k+1}\right)-\cos \left(u^{k}\right)}{u^{k+1}-u^{k}}\right)\right| \\
& \leqslant\left|\left(\frac{\cos \left(u_{e}^{k+1}\right)-\cos \left(u_{e}^{k}\right)}{u_{e}^{k+1}-u_{e}^{k}}-\frac{\cos \left(u^{k+1}\right)-\cos \left(u_{e}^{k}\right)}{u^{k+1}-u_{e}^{k}}\right)\right| \\
& \quad+\left|\left(\frac{\cos \left(u^{k+1}\right)-\cos \left(u_{e}^{k}\right)}{u^{k+1}-u_{e}^{k}}-\frac{\cos \left(u^{k+1}\right)-\cos \left(u^{k}\right)}{u^{k+1}-u^{k}}\right)\right|
\end{aligned}
$$

and recalling the definition (3.4) of $h(x)$, we have the following results

$$
\begin{aligned}
\left|\left(\frac{\cos \left(u_{e}^{k+1}\right)-\cos \left(u_{e}^{k}\right)}{u_{e}^{k+1}-u_{e}^{k}}-\frac{\cos \left(u^{k+1}\right)-\cos \left(u_{e}^{k}\right)}{u^{k+1}-u_{e}^{k}}\right)\right| & \leqslant\left|h\left(u_{e}^{k+1}\right)-h\left(u^{k+1}\right)\right| \\
& =\left|h^{\prime}\left(\eta_{1}\right)\right| \cdot\left|u_{e}^{k+1}-u^{k+1}\right| \leqslant\left|\tilde{u}^{k+1}\right|,
\end{aligned}
$$


in which we choose $a=u_{e}^{k}$, and

$$
\begin{aligned}
\left|\left(\frac{\cos \left(u^{k+1}\right)-\cos \left(u_{e}^{k}\right)}{u^{k+1}-u_{e}^{k}}-\frac{\cos \left(u^{k+1}\right)-\cos \left(u^{k}\right)}{u^{k+1}-u^{k}}\right)\right| & \leqslant\left|h\left(u_{e}^{k}\right)-h\left(u^{k}\right)\right| \\
& =\left|h^{\prime}\left(\eta_{2}\right)\right| \cdot\left|u_{e}^{k}-u^{k}\right| \leqslant\left|\tilde{u}^{k}\right|,
\end{aligned}
$$

in which $a=u^{k+1}$, respectively. Substituting (4.7) and (4.8) into (4.6) gives

$$
\left|\left(\frac{\cos \left(u_{e}^{k+1}\right)-\cos \left(u_{e}^{k}\right)}{u_{e}^{k+1}-u_{e}^{k}}-\frac{\cos \left(u^{k+1}\right)-\cos \left(u^{k}\right)}{u^{k+1}-u^{k}}\right)\right| \leqslant\left|\tilde{u}^{k+1}\right|+\left|\tilde{u}^{k}\right| .
$$

Also, it follows from (4.4) that

$$
\tilde{u}^{k+1}-\tilde{u}^{k}=\frac{\Delta t}{2}\left(\tilde{v}^{k+1}+\tilde{v}^{k}\right)+\Delta t s^{k}
$$

Therefore, we can obtain the estimate as follows,

$$
\begin{aligned}
& \left\langle\phi\left(\frac{\cos \left(u_{e}^{k+1}\right)-\cos \left(u_{e}^{k}\right)}{u_{e}^{k+1}-u_{e}^{k}}-\frac{\cos \left(u^{k+1}\right)-\cos \left(u^{k}\right)}{u^{k+1}-u^{k}}\right), \tilde{u}^{k+1}-\tilde{u}^{k}\right\rangle \\
& \leqslant \phi_{0}\left\langle\left|\tilde{u}^{k+1}\right|+\left|\tilde{u}^{k}\right|,\left|\tilde{u}^{k+1}-\tilde{u}^{k}\right|\right\rangle \\
& \leqslant \frac{\phi_{0}}{2} \Delta \mathrm{t}\left\langle\left|\tilde{u}^{k+1}\right|+\left|\tilde{u}^{k}\right|,\left|\tilde{v}^{k+1}\right|+\left|\tilde{v}^{k}\right|\right\rangle+\phi_{0} \Delta t\left\langle\left|\tilde{u}^{k+1}\right|+\left|\tilde{u}^{k}\right|,\left|s^{k}\right|\right\rangle \\
& \leqslant \frac{\phi_{0}}{4} \Delta \mathrm{t}\left(\left\|\left(\left|\tilde{u}^{k+1}\right|+\left|\tilde{u}^{k}\right|\right)\right\|_{2}^{2}+\left\|\left(\left|\tilde{v}^{k+1}\right|+\left|\tilde{v}^{k}\right|\right)\right\|_{2}^{2}\right)+\frac{\phi_{0}}{2} \Delta \mathrm{t}\left(\left\|\left(\left|\tilde{u}^{k+1}\right|_{2}^{2}+\left|\tilde{u}^{k}\right|\right)\right\|_{2}^{2}\right)+\phi_{0} \Delta t\left\|s^{k}\right\|_{2}^{2} \\
& \leqslant \frac{\phi_{0}}{2} \Delta \mathrm{t}\left(\left\|\tilde{u}^{k+1}\right\|_{2}^{2}+\left\|\tilde{u}^{k}\right\|_{2}^{2}+\left\|\tilde{v}^{k+1}\right\|_{2}^{2}+\left\|\tilde{v}^{k}\right\|_{2}^{2}\right)+\phi_{0} \Delta \mathrm{t}\left(\left\|\tilde{u}^{k+1}\right\|_{2}^{2}+\left\|\tilde{u}^{k}\right\|_{2}^{2}\right)+\phi_{0} \Delta t\left\|s^{k}\right\|_{2}^{2} \\
& \leqslant \frac{3 \phi_{0}}{2} \Delta \mathrm{t}\left(\left\|\tilde{u}^{k+1}\right\|_{2}^{2}+\left\|\tilde{u}^{k}\right\|_{2}^{2}\right)+\frac{\phi_{0}}{2} \Delta \mathrm{t}\left(\left\|\tilde{v}^{k+1}\right\|_{2}^{2}+\left\|\tilde{v}^{k}\right\|_{2}^{2}\right)+\phi_{0} \Delta \mathrm{t}\left\|s^{k}\right\|_{2}^{2},
\end{aligned}
$$

where the inequality $(a+b)^{2} \leqslant 2\left(a^{2}+b^{2}\right)$ is used.

For the first term in (4.5), using (4.4), we can arrive at,

$$
\begin{aligned}
\frac{1}{\Delta \mathrm{t}}\left\langle\tilde{v}^{\mathrm{k}+1}-\tilde{v}^{\mathrm{k}}, \tilde{\mathrm{u}}^{\mathrm{k}+1}-\tilde{\mathrm{u}}^{\mathrm{k}}\right\rangle & =\frac{1}{2}\left\langle\tilde{v}^{\mathrm{k}+1}-\tilde{v}^{\mathrm{k}}, \tilde{v}^{\mathrm{k}+1}+\tilde{v}^{\mathrm{k}}\right\rangle+\Delta \mathrm{t}\left\langle\tilde{v}^{\mathrm{k}+1}-\tilde{v}^{\mathrm{k}}, \mathrm{s}^{\mathrm{k}}\right\rangle \\
& =\frac{1}{2}\left(\left\|\tilde{v}^{\mathrm{k}+1}\right\|_{2}^{2}-\left\|\tilde{v}^{\mathrm{k}}\right\|_{2}^{2}\right)+\Delta \mathrm{t}\left\langle\tilde{v}^{\mathrm{k}+1}-\tilde{v}^{\mathrm{k}}, \mathrm{s}^{\mathrm{k}}\right\rangle \\
& \geqslant \frac{1}{2}\left(\left\|\tilde{v}^{\mathrm{k}+1}\right\|_{2}^{2}-\left\|\tilde{v}^{\mathrm{k}}\right\|_{2}^{2}\right)-\frac{1}{2} \Delta \mathrm{t}\left(\left\|\tilde{v}^{\mathrm{k}+1}-\tilde{v}^{\mathrm{k}}\right\|_{2}^{2}+\left\|\mathrm{s}^{\mathrm{k}}\right\|_{2}^{2}\right) \\
& \geqslant \frac{1}{2}\left(\left\|\tilde{v}^{\mathrm{k}+1}\right\|_{2}^{2}-\left\|\tilde{v}^{\mathrm{k}}\right\|_{2}^{2}\right)-\Delta \mathrm{t}\left(\left\|\tilde{v}^{\mathrm{k}+1}\right\|_{2}^{2}+\left\|\tilde{v}^{\mathrm{k}}\right\|_{2}^{2}\right)-\frac{1}{2} \Delta \mathrm{t}\left\|\mathrm{s}^{\mathrm{k}}\right\|_{2}^{2}
\end{aligned}
$$

And it follows from the second term in (4.5) that

$$
\frac{\beta}{\Delta t}\left\langle\tilde{u}^{k+1}-\tilde{u}^{k}, \tilde{u}^{k+1}-\tilde{u}^{k}\right\rangle=\frac{\beta}{\Delta t}\left\|\tilde{u}^{k+1}-\tilde{u}^{k}\right\|_{2}^{2} .
$$

As for the diffusion term, the following result is also straightforward from (2.7),

$$
-\left\langle\Delta_{h}\left(\tilde{u}^{k+1}+\tilde{u}^{k}\right), \tilde{u}^{k+1}-\tilde{u}^{k}\right\rangle=\left\langle\nabla_{h}\left(\tilde{u}^{k+1}+\tilde{u}^{k}\right), \nabla_{h}\left(\tilde{u}^{k+1}-\tilde{u}^{k}\right)\right\rangle,=\left\|\nabla_{h} \tilde{u}^{k+1}\right\|_{2}^{2}-\left\|\nabla_{h} \tilde{u}^{k}\right\|_{2}^{2} .
$$

In turn, considering the local truncation error term in (4.5), we have

$$
\begin{aligned}
\left\langle\rho^{\mathrm{k}}, \tilde{\mathrm{u}}^{\mathrm{k}+1}-\tilde{\mathrm{u}}^{\mathrm{k}}\right\rangle=\Delta \mathrm{t}\left\langle\rho^{\mathrm{k}}, \frac{\tilde{\mathrm{u}}^{\mathrm{k}+1}-\tilde{\mathrm{u}}^{\mathrm{k}}}{\Delta \mathrm{t}}\right\rangle & =\frac{1}{2} \Delta \mathrm{t}\left\langle\rho^{\mathrm{k}}, \tilde{v}^{\mathrm{k}+1}+\tilde{v}^{\mathrm{k}}\right\rangle+\Delta \mathrm{t}\left\langle\rho^{\mathrm{k}}, \mathrm{s}^{\mathrm{k}}\right\rangle \\
& \leqslant \frac{1}{4} \Delta \mathrm{t}\left(\left\|\rho^{\mathrm{k}}\right\|_{2}^{2}+\left\|\tilde{v}^{\mathrm{k}+1}+\tilde{v}^{\mathrm{k}}\right\|_{2}^{2}\right)+\frac{1}{2} \Delta \mathrm{t}\left(\left\|\rho^{\mathrm{k}}\right\|_{2}^{2}+\left\|\mathrm{s}^{\mathrm{k}}\right\|_{2}^{2}\right) \\
& \leqslant \frac{3}{4} \Delta \mathrm{t}\left\|\rho^{\mathrm{k}}\right\|_{2}^{2}+\frac{1}{2} \Delta \mathrm{t}\left\|\mathrm{s}^{\mathrm{k}}\right\|_{2}^{2}+\frac{1}{2} \Delta \mathrm{t}\left(\left\|\tilde{v}^{\mathrm{k}+1}\right\|_{2}^{2}+\left\|\tilde{v}^{\mathrm{k}}\right\|_{2}^{2}\right) .
\end{aligned}
$$


Consequently, it follows from (4.5), (4.9), (4.10), (4.11), and (4.12) that

$$
\begin{aligned}
& \frac{1}{2}\left(\left\|\tilde{v}^{k+1}\right\|_{2}^{2}-\left\|\tilde{v}^{k}\right\|_{2}^{2}\right)+\frac{\alpha}{2}\left(\left\|\nabla_{h} \tilde{u}^{k+1}\right\|_{2}^{2}-\left\|\nabla_{h} \tilde{u}^{k}\right\|_{2}^{2}\right)+\frac{\beta}{\Delta t}\left\|\tilde{u}^{k+1}-\tilde{u}^{k}\right\|_{2}^{2} \\
& \quad \leqslant \frac{3}{2} \phi_{0} \Delta t\left(\left\|\tilde{u}^{k+1}\right\|_{2}^{2}+\left\|\tilde{u}^{k}\right\|_{2}^{2}\right)+\left(\frac{3}{2}+\frac{\phi_{0}}{2}\right) \Delta t\left(\left\|\tilde{v}^{k+1}\right\|_{2}^{2}+\left\|\tilde{v}^{k}\right\|_{2}^{2}\right)+\frac{3}{4} \Delta t\left\|\rho^{k}\right\|_{2}^{2}+\left(\phi_{0}+1\right) \Delta t\left\|s^{k}\right\|_{2}^{2} .
\end{aligned}
$$

Unfortunately, we so far can not obtain the estimates for $\left\|\tilde{u}^{k}\right\|$ and $\left\|\tilde{v}^{k}\right\|$ by the discrete Gronwall inequality, since the term $\left\|\tilde{u}^{k}\right\|$ does not appear in the left-hand part of (4.13). To remedy this, we need to analyze the estimate for $\left\|\tilde{u}^{k}\right\|$.

Going back to (4.4), it can be rewritten as

$$
\tilde{\mathrm{u}}^{\mathrm{k}}-\tilde{\mathrm{u}}^{\mathrm{k}-1}=\frac{1}{2} \Delta \mathrm{t}\left(\tilde{v}^{\mathrm{k}}+\tilde{v}^{\mathrm{k}-1}\right)+\Delta \mathrm{ts} \mathrm{s}^{\mathrm{k}-1} .
$$

Summing up (4.14) from 1 to $k$, we get

$$
\tilde{u}^{k}=\tilde{u}^{0}+\frac{1}{2} \Delta t \sum_{l=1}^{k}\left(\tilde{v}^{l}+\tilde{v}^{l-1}\right)+\Delta t \sum_{l=1}^{k-1} s^{l}=\frac{1}{2} \Delta t \sum_{l=1}^{k}\left(\tilde{v}^{l}+\tilde{v}^{l-1}\right)+\Delta t \sum_{l=1}^{k-1} s^{l},
$$

where we apply the fact that $\tilde{u}^{0}=0$. In turn, an application of Cauchy inequality implies that

$$
\begin{aligned}
\left|\tilde{u}^{k}\right|^{2} & \leqslant 2\left[\frac{1}{4} \Delta \mathrm{t}^{2}\left(\sum_{l=1}^{\mathrm{k}}\left(\tilde{v}^{\mathrm{l}}+\tilde{v}^{\mathrm{l}-1}\right)\right)^{2}+\Delta \mathrm{t}^{2}\left(\sum_{\mathrm{l}=1}^{\mathrm{k}-1} s^{\mathrm{l}}\right)^{2}\right] \\
& \leqslant \frac{1}{2} \Delta \mathrm{t}^{2} \sum_{\mathrm{l}=1}^{\mathrm{k}} 2\left(\left|\tilde{v}^{\mathrm{l}}\right|^{2}+\left|\tilde{v}^{\mathrm{l}-1}\right|^{2}\right)+\Delta \mathrm{t}^{2}\left(\sum_{\mathrm{l}=1}^{\mathrm{k}-1} \mathrm{O}\left(\Delta \mathrm{t}^{2}\right)\right)^{2} \\
& \leqslant 2 \mathrm{k} \Delta \mathrm{t}^{2} \sum_{\mathrm{l}=1}^{\mathrm{k}}\left|\tilde{v}^{\mathrm{l}}\right|^{2}+\mathrm{k}^{2} \Delta \mathrm{t}^{2}\left(\mathrm{O}\left(\Delta \mathrm{t}^{2}\right)\right)^{2} \\
& \leqslant 2 \mathrm{~T} \Delta \mathrm{t} \sum_{\mathrm{l}=1}^{\mathrm{k}}\left|\tilde{v}^{\mathrm{l}}\right|^{2}+\mathrm{T}^{2} \mathrm{O}\left(\Delta \mathrm{t}^{4}\right)
\end{aligned}
$$

in which $k \Delta t \leqslant T$ is used in the last step. This shows

$$
\left|\tilde{u}^{k}\right|^{2} \leqslant 2 \mathrm{~T} \Delta \mathrm{t} \sum_{\mathrm{l}=1}^{\mathrm{k}}\left|\tilde{v}^{\mathrm{l}}\right|^{2}+\mathrm{C} \cdot \mathrm{O}\left(\Delta \mathrm{t}^{4}\right), \quad\left|\tilde{u}^{\mathrm{k}+1}\right|^{2} \leqslant 2 \mathrm{~T} \Delta \mathrm{t} \sum_{\mathrm{l}=1}^{\mathrm{k}+1}\left|\tilde{v}^{\mathrm{l}}\right|^{2}+\mathrm{C} \cdot \mathrm{O}\left(\Delta \mathrm{t}^{4}\right) .
$$

Substituting the result above into (4.13) leads to

$$
\begin{aligned}
& \frac{1}{2}\left(\left\|\tilde{v}^{k+1}\right\|_{2}^{2}-\left\|\tilde{v}^{k}\right\|_{2}^{2}\right)+\frac{\alpha}{2}\left(\left\|\nabla_{h} \tilde{u}^{k+1}\right\|_{2}^{2}-\left\|\nabla_{h} \tilde{u}^{k}\right\|_{2}^{2}\right)+\frac{\beta}{\Delta t}\left\|\tilde{u}^{k+1}-\tilde{u}^{k}\right\|_{2}^{2} \\
& \leqslant 6 \phi_{0} \mathrm{~T} \Delta \mathrm{t}^{2} \sum_{l=0}^{k+1}\left\|\tilde{v}^{\mathrm{l}}\right\|_{2}^{2}+\left(\frac{3}{2}+\frac{\phi_{0}}{2}\right) \Delta \mathrm{t}\left(\left\|\tilde{v}^{k+1}\right\|_{2}^{2}+\left\|\tilde{v}^{k}\right\|_{2}^{2}\right)+\mathrm{C} \Delta \mathrm{t} \cdot \mathrm{O}\left(\Delta \mathrm{t}^{4}+\mathrm{h}^{4}\right) .
\end{aligned}
$$

Summing over $\mathrm{k}$ from 0 to $\mathrm{n}-1$, and using $\tilde{\mathrm{u}}^{0}=0, \tilde{v}^{0}=0$, we have

$$
\frac{1}{2}\left\|\tilde{v}^{n}\right\|_{2}^{2}+\frac{\alpha}{2}\left\|\nabla_{h} \tilde{u}^{n}\right\|_{2}^{2} \leqslant 6 \phi_{0} \mathrm{~T} \Delta \mathrm{t}^{2} \sum_{k=0}^{n-1} \sum_{l=0}^{k+1}\left\|\tilde{v}^{l}\right\|_{2}^{2}+\left(3+\phi_{0}\right) \Delta \mathrm{t} \sum_{k=0}^{n-1}\left\|\tilde{v}^{k}\right\|_{2}^{2}+\mathrm{CT} \cdot \mathrm{O}\left(\Delta \mathrm{t}^{4}+\mathrm{h}^{4}\right) .
$$


Then according to $\sum_{l=0}^{k+1}\left\|\tilde{v}^{l}\right\|_{2}^{2} \leqslant \sum_{l=0}^{n}\left\|\tilde{v}^{l}\right\|_{2}^{2}$ for $0 \leqslant k+1 \leqslant n$, the inequality above can be rewritten as

$$
\frac{1}{2}\left\|\tilde{v}^{n}\right\|_{2}^{2}+\frac{\alpha}{2}\left\|\nabla_{h} \tilde{u}^{n}\right\|_{2}^{2} \leqslant\left(6 \phi_{0} T^{2}+3+\phi_{0}\right) \Delta t \sum_{k=0}^{n}\left\|\tilde{v}^{k}\right\|_{2}^{2}+C T \cdot O\left(\Delta t^{4}+h^{4}\right) .
$$

Let $\tilde{E}^{n}=\left\|\tilde{v}^{n}\right\|_{2}^{2}+\alpha\left\|\nabla_{h} \tilde{u}^{n}\right\|_{2}^{2}$. Then we get

$$
\tilde{\mathrm{E}}^{n} \leqslant\left(12 \phi_{0} \mathrm{~T}^{2}+6+2 \phi_{0}\right) \Delta \mathrm{t} \sum_{\mathrm{l}=0}^{\mathrm{n}-1} \tilde{\mathrm{E}}^{\mathrm{l}}+\mathrm{CT} \cdot \mathrm{O}\left(\Delta \mathrm{t}^{4}+\mathrm{h}^{4}\right) .
$$

By the discrete Gronwall inequality, we derive that

$$
\left\|\tilde{v}^{\mathrm{n}}\right\|_{2}^{2}+\alpha\left\|\nabla_{\mathrm{h}} \tilde{u}^{\mathrm{n}}\right\|_{2}^{2} \leqslant \tilde{\mathrm{C}} \cdot \mathrm{O}\left(\Delta \mathrm{t}^{4}+\mathrm{h}^{4}\right),
$$

that is,

$$
\left\|\tilde{v}^{\mathfrak{n}}\right\|_{2}+\alpha\left\|\nabla_{h} \tilde{u}^{\mathfrak{n}}\right\|_{2} \leqslant \tilde{C} \cdot O\left(\Delta t^{2}+h^{2}\right),
$$

where $\tilde{C}$ is independent on $\Delta t$ and $h$. Moreover, from (4.15), we have

$$
\left|\tilde{u}^{\mathrm{n}}\right|^{2} \leqslant 2 \mathrm{~T} \Delta \mathrm{t} \sum_{\mathrm{l}=1}^{\mathrm{k}}\left|\tilde{v}^{\mathrm{l}}\right|^{2}+\mathrm{C} \cdot \mathrm{O}\left(\Delta \mathrm{t}^{4}\right) \leqslant \mathrm{C} \cdot \mathrm{O}\left(\Delta \mathrm{t}^{4}+\mathrm{h}^{4}\right) .
$$

Finally, the combination of (4.16) and (4.17) gives

$$
\left\|\tilde{v}^{\mathfrak{n}}\right\|_{2}+\left\|\tilde{u}^{\mathfrak{n}}\right\|_{2}+\alpha\left\|\nabla_{\mathrm{h}} \tilde{\mathrm{u}}^{\mathrm{n}}\right\|_{2} \leqslant \tilde{\mathrm{C}} \cdot \mathrm{O}\left(\Delta \mathrm{t}^{2}+\mathrm{h}^{2}\right),
$$

which shows the unconditional convergence in the sense of $l^{2}$-norm is obtained. This completes the proof of Theorem 4.2.

Remark 4.3. For the one dimensional sine-Gordon equation, by the discrete version of the Sobolev imbedding inequality, the $\|\cdot\|_{\infty}$ estimate can be obtained from the conservative property and the $l^{2}$-norm. But for two dimension case, $\|\cdot\|_{\infty}$ error bounds of the numerical scheme (2.1) and (2.2) maybe slightly complicated in obtaining a priori uniform estimate of the numerical solution. It also can be obtained if we perform a higher consistency analysis by a careful Taylor expansion. The details are skipped for simplicity of presentation and an analogous technique can be seen in [18].

\section{Numerical simulations}

We now perform a couple of numerical experiments that support the theoretical results and error estimates for the scheme given by (2.3)-(2.4).

\subsection{Verification of the second order accuracy}

In the first test, we consider the equation (1.1) in the domain $\Omega=\left[-\frac{1}{2}, \frac{1}{2}\right] \times\left[-\frac{1}{2}, \frac{1}{2}\right]$ with $\beta=0$, $\phi(x, y)=1, \alpha=\frac{1}{2 \pi^{2}}$, and $f(x, y, t)=\sin (\cos (\pi x) \cos (\pi y) \cos (t))$. The exact solution of (1.1) is given by

$$
u_{e}(x, y, t)=\cos (\pi x) \cos (\pi y) \cos (t), \quad v_{e}(x, y, t)=-\cos (\pi x) \cos (\pi y) \sin (t) .
$$

The initial conditions and the boundary condition can be obtained from the exact solution. Fig. 1 and Fig. 2 show the profile of the exact solution $u$ at $t=0$ on $\Omega$, and the numerical solutions $u^{n}$ at time $\mathrm{T}=0.8,1.5,3$ with $\Delta \mathrm{t}=0.1$ and $\mathrm{h}=0.025$, respectively. The errors in the sense of $l^{2}$-norm of the numerical solutions for different mesh steps $h$ and $\Delta t$ at times $t=1,2,3,4$, and 5 can be found in Table 1 , and the corresponding numerical orders of convergence are listed in Table 2. Clearly, it verifies the second order accuracy in Theorem 4.2. 

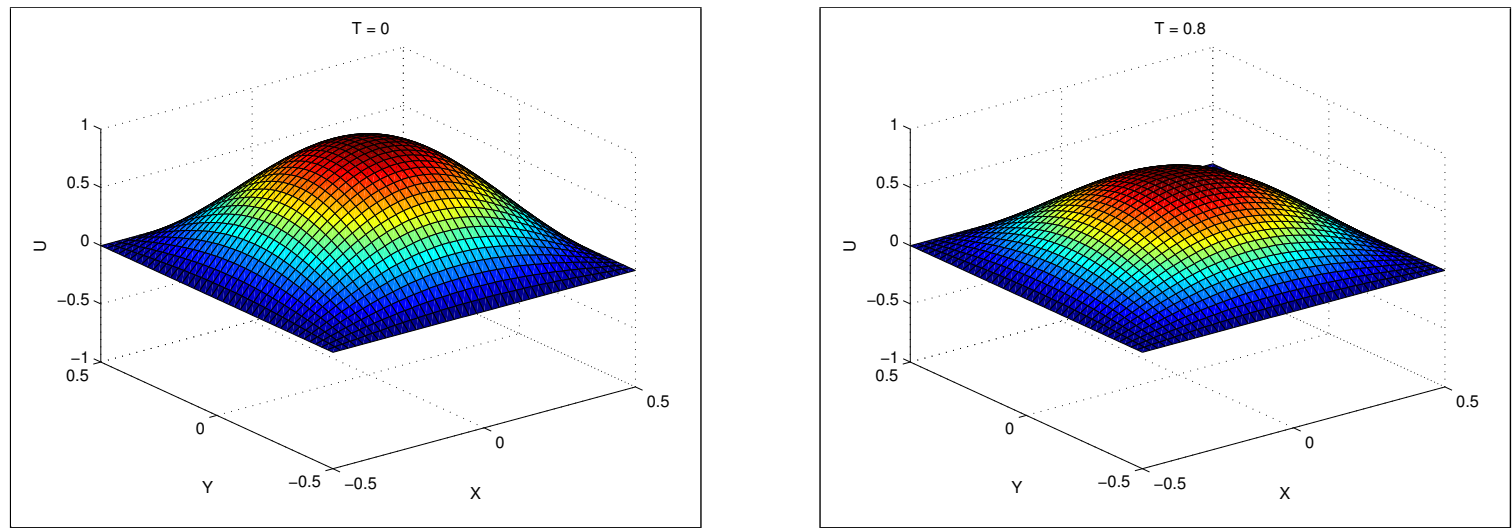

Figure 1: Left: Profile of the exact solution $u(x, y, t)$ at $t=0$. Right: Numerical solutions of $u$ at $T=0.8$ with $h=0.025$ and $\Delta \mathrm{t}=0.1$.
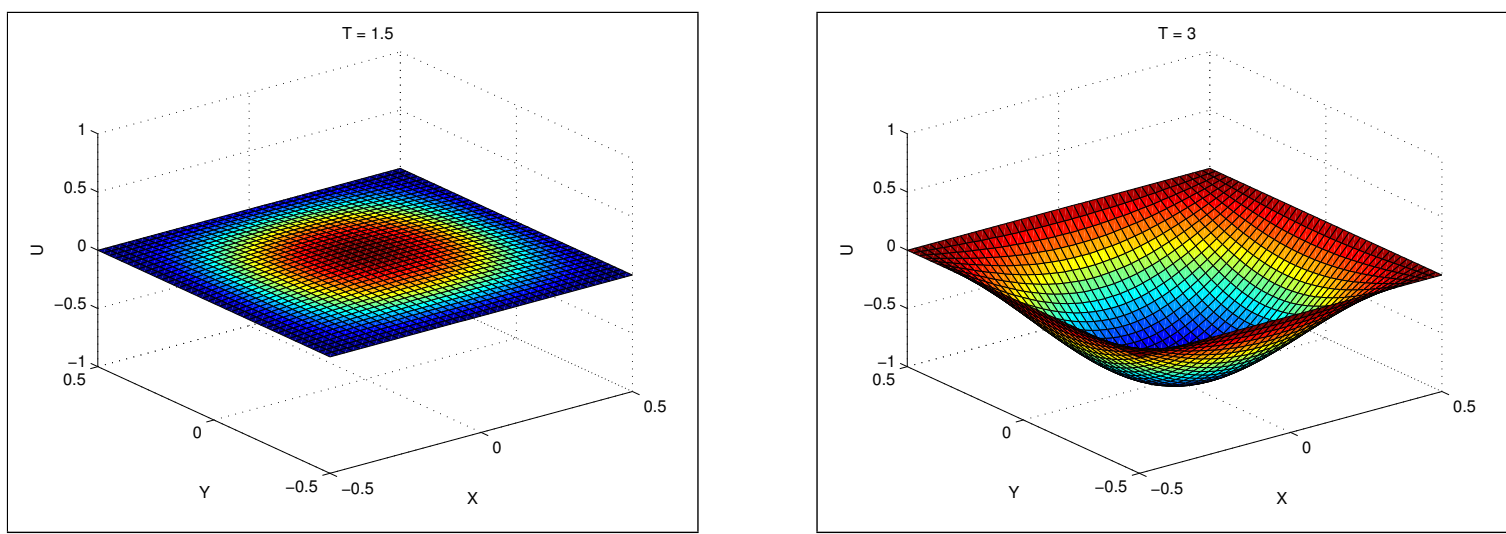

Figure 2: Left: Numerical solutions of $u$ at $T=1.5$ with $h=0.025$ and $\Delta t=0.1$. Right: Numerical solutions of $u$ at $T=3$ with $h=0.025$ and $\Delta t=0.1$.

Table 1: The $\|\cdot\|_{2}$ errors estimates of $\mathrm{u}^{\mathrm{n}}$ and $v^{\mathrm{n}}$ with various values $\mathrm{h}$ and $\Delta \mathrm{t}$.

\begin{tabular}{ccccccc}
\hline & \multicolumn{2}{c}{$\Delta \mathrm{t}=0.2, \mathrm{~h}=0.1$} & \multicolumn{2}{c}{$\Delta \mathrm{t}=0.1, \mathrm{~h}=0.05$} & \multicolumn{2}{c}{$\Delta \mathrm{t}=0.05, \mathrm{~h}=0.025$} \\
\hline & $\left\|\tilde{\mathrm{u}}^{\mathrm{n}}\right\|_{2}$ & $\left\|\tilde{v}^{\mathfrak{n}}\right\|_{2}$ & $\left\|\tilde{\mathrm{u}}^{\mathrm{n}}\right\|_{2}$ & $\left\|\tilde{v}^{\mathfrak{n}}\right\|_{2}$ & $\left\|\tilde{\mathrm{u}}^{\mathrm{n}}\right\|_{2}$ & $\left\|\tilde{v}^{\mathrm{n}}\right\|_{2}$ \\
\hline $\mathrm{t}=1$ & $3.6332417 \mathrm{e}-3$ & $4.10777448 \mathrm{e}-3$ & $9.1807718 \mathrm{e}-4$ & $1.0275471 \mathrm{e}-3$ & $2.3013872 \mathrm{e}-4$ & $2.5692078 \mathrm{e}-4$ \\
$\mathrm{t}=2$ & $5.3736869 \mathrm{e}-3$ & $5.7848266 \mathrm{e}-3$ & $1.3423090 \mathrm{e}-3$ & $1.4826793 \mathrm{e}-3$ & $3.3549256 \mathrm{e}-4$ & $3.7298456 \mathrm{e}-4$ \\
$\mathrm{t}=3$ & $4.8006248 \mathrm{e}-3$ & $1.4044778 \mathrm{e}-2$ & $1.2425939 \mathrm{e}-3$ & $3.5219515 \mathrm{e}-3$ & $3.1334248 \mathrm{e}-4$ & $8.8112076 \mathrm{e}-4$ \\
$\mathrm{t}=4$ & $1.5080860 \mathrm{e}-2$ & $1.8066820 \mathrm{e}-3$ & $3.7880320 \mathrm{e}-3$ & $3.6020681 \mathrm{e}-4$ & $9.4806730 \mathrm{e}-4$ & $8.4218108 \mathrm{e}-5$ \\
$\mathrm{t}=5$ & $6.3731025 \mathrm{e}-3$ & $2.1098154 \mathrm{e}-2$ & $1.5032623 \mathrm{e}-3$ & $5.3465660 \mathrm{e}-3$ & $3.7005127 \mathrm{e}-4$ & $1.3410308 \mathrm{e}-3$ \\
\hline
\end{tabular}

\subsection{Energy conservation for the undamped equation}

In the second test, we consider the homogeneous boundary condition $\left.u\right|_{\partial \Omega}=0$ for the equation (1.4) on $\Omega=[0,1] \times[0,1]$ with $\phi=1$,

$$
\varphi_{1}(x, y)=\sin (2 \pi x) \sin (2 \pi y), \quad \varphi_{2}(x, y)=0 .
$$

We take $T=1$ and the discrete energy $\mathcal{E}^{n}$ at the different time for $\Delta t=0.002, h=0.05$ and $\Delta t=0.001, h=$ 0.025 can be found in Table 3. Obviously, values of $\mathcal{E}^{n}$ at the different time remain nearly a constant as time increases. 
Table 2: Numerical verification of theoretical accuracy $\mathrm{O}\left(\Delta \mathrm{t}^{2}+\mathrm{h}^{2}\right)$.

\begin{tabular}{ccccccc}
\hline & $\left\|\tilde{\mathrm{u}}^{\mathrm{n}}(\mathrm{h}, \Delta \mathrm{t})\right\|_{2} /\left\|\tilde{\mathrm{u}}^{2 \mathrm{n}}\left(\frac{\mathrm{h}}{2}, \frac{\Delta \mathrm{t}}{2}\right)\right\|_{2}$ & \multicolumn{3}{c}{$\left\|\tilde{v}^{\mathrm{n}}(\mathrm{h}, \Delta \mathrm{t})\right\|_{2} /\left\|\tilde{v}^{2 \mathfrak{n}}\left(\frac{\mathrm{h}}{2}, \frac{\Delta \mathrm{t}}{2}\right)\right\|_{2}$} \\
\hline & $\Delta \mathrm{t}=0.2$ & $\Delta \mathrm{t}=0.1$ & $\Delta \mathrm{t}=0.05$ & $\Delta \mathrm{t}=0.2$ & $\Delta \mathrm{t}=0.1$ & $\Delta \mathrm{t}=0.05$ \\
& $\mathrm{~h}=0.1$ & $\mathrm{~h}=0.05$ & $\mathrm{~h}=0.025$ & $\mathrm{~h}=0.1$ & $\mathrm{~h}=0.05$ & $\mathrm{~h}=0.025$ \\
\hline $\mathrm{t}=1$ & - & 1.9846 & 1.9961 & - & 1.9992 & 1.9998 \\
$\mathrm{t}=2$ & - & 2.0012 & 2.0004 & - & 1.9641 & 1.9910 \\
$\mathrm{t}=3$ & - & 1.9499 & 1.9875 & - & 1.9956 & 1.9990 \\
$\mathrm{t}=4$ & - & 1.9932 & 1.9984 & - & 2.3264 & 2.0966 \\
$\mathrm{t}=5$ & - & 2.0839 & 2.0223 & - & 1.9804 & 1.9953 \\
\hline
\end{tabular}

Table 3: The discrete energy $\mathcal{E}^{n}$ with different $\Delta t$ and $h$.

\begin{tabular}{ccc}
\hline & $\Delta \mathrm{t}=0.002, \mathrm{~h}=0.05$ & $\Delta \mathrm{t}=0.001, \mathrm{~h}=0.025$ \\
\hline $\mathrm{t}=0.2$ & 10.351697 & 10.087760 \\
$\mathrm{t}=0.4$ & 10.216081 & 10.031903 \\
$\mathrm{t}=0.6$ & 10.151906 & 10.112902 \\
$\mathrm{t}=0.8$ & 10.288459 & 10.199227 \\
$\mathrm{t}=1$ & 10.419025 & 10.280056 \\
\hline
\end{tabular}

\subsection{Circular ring soliton}

The behavior of a circular ring quasi-soliton arising from the sine-Gordon equation is named as waves pulsons because of their pulsating behavior. In this test, we consider the equation (1.1) on $\Omega=[-4,4] \times$ $[-4,4]$ with $\phi(x, y)=1$. The initial conditions are given by,

$$
\varphi_{1}(x, y)=2 \arctan \left(\exp \left(3-5 \sqrt{x^{2}+y^{2}}\right)\right), \quad \varphi_{2}(x, y)=0,
$$

and the boundary condition is periodic. Similar to [2], in order to study the evolution of the ring solitons, we plot both the surfaces and the corresponding contours in terms of $\sin \left(\frac{\mathfrak{u}}{2}\right)$ with $h=0.1$ and $\Delta t=0.1$. As seen from Figs. 3, 4, 5, and 6, ring soliton shrinks for initial stage $(t=0)$, but as time goes on, oscillations and radiations begin to form and continue to form up to $t=4$. At $t=6$, the graph shows that a ring soliton is nearly formed again. These graphs are consistent with earlier work on this topic in $[2,14]$. Furthermore, with the implicit treatment and the linear iteration algorithm, it becomes possible to simulate the long time behaviors for such an equation. In Fig. 7, the profile of the numerical solutions of $\mathrm{u}$ with $\mathrm{h}=0.1$ and $\Delta \mathrm{t}=0.1$ at $\mathrm{T}=50$ is presented.
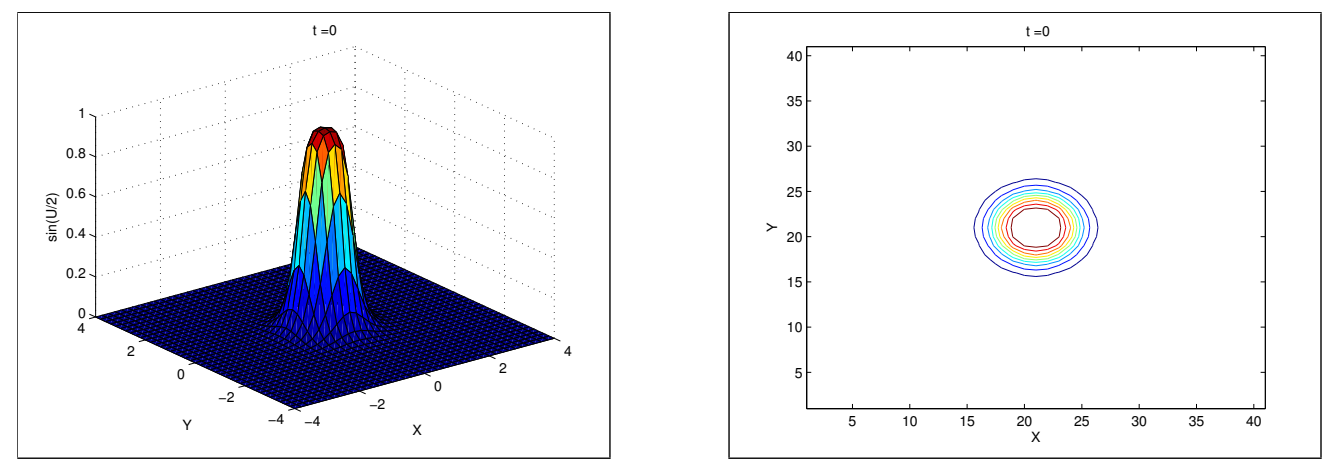

Figure 3: The initial function of $u$ and its contour profile. 

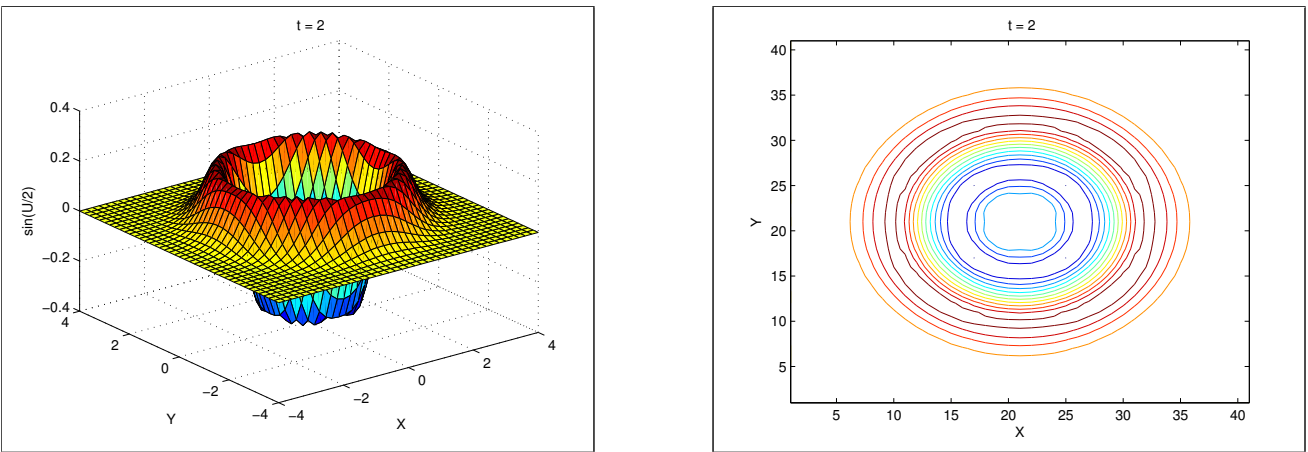

Figure 4: Numerical solutions of $u$ and the contour profile at $T=2$ with $h=0.1$ and $\Delta t=0.1$.
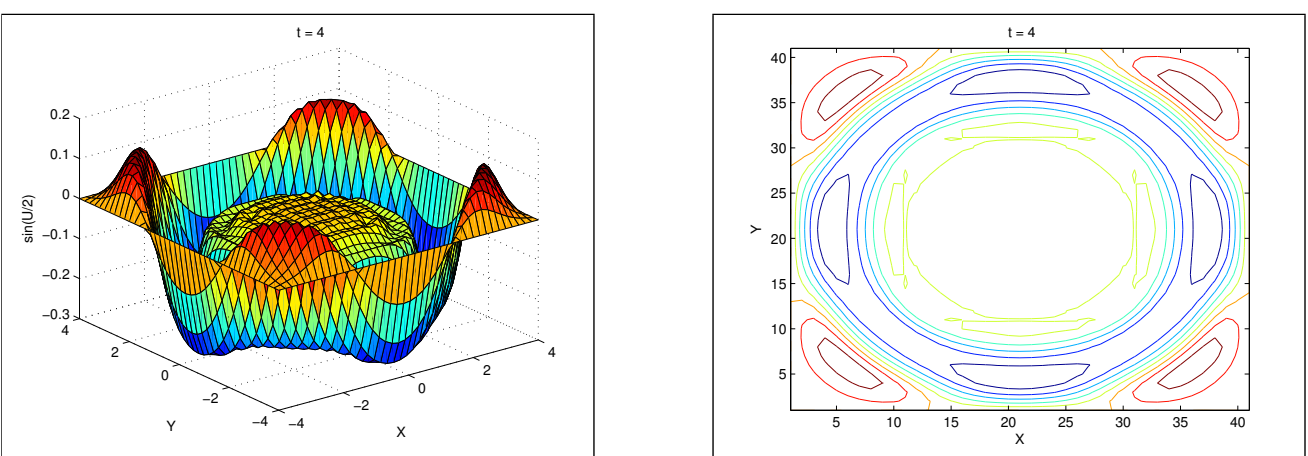

Figure 5: Numerical solutions of $u$ and the contour profile at $\mathrm{T}=4$ with $\mathrm{h}=0.1$ and $\Delta \mathrm{t}=0.1$.
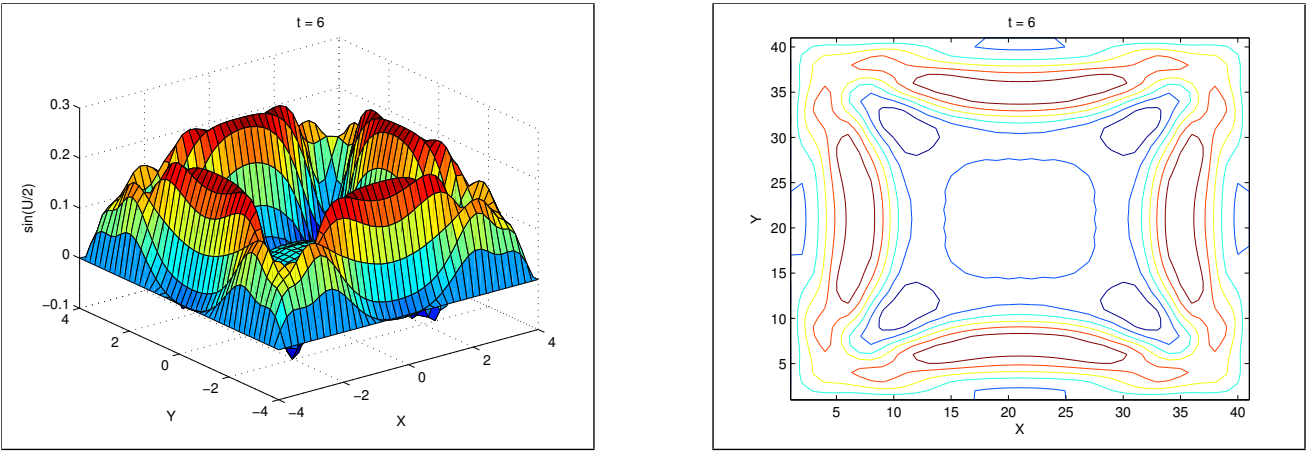

Figure 6: Numerical solutions of $u$ and the contour profile at $T=6$ with $h=0.1$ and $\Delta t=0.1$.
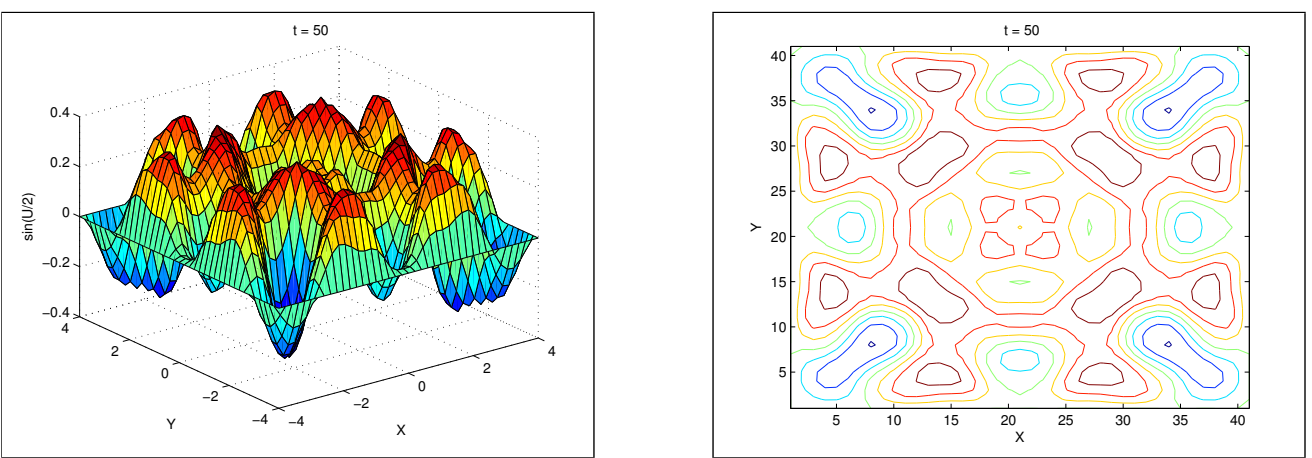

Figure 7: Numerical solutions of $u$ and the contour profile at $T=50$ with $h=0.1$ and $\Delta t=0.1$. 


\section{Conclusion}

In this paper, we discussed a second-order semi-implicit finite difference scheme for the 2D sineGordon equation, which can admit the discrete energy conservation for the undamped problem. We also proposed the efficient linear iteration algorithm for approximating the nonlinear system arising from the implicit treatment of the nonlinear term. Moreover, the iteration algorithm was proven to be a contraction mapping. In turn, based on truncation errors, the convergence analysis of the numerical scheme was also shown. Furthermore, the results of numerical experiments demonstrated the efficiency and the accuracy of our proposed scheme.

\section{Acknowledgment}

The authors are very grateful to reviewers for carefully reading this paper and their comments. We appreciate the support provided for this paper by the Science and Technology Department of Sichuan Province in China (No. 2017GZ0316), the funds of Sichuan Center for Education Development Research of Education Department (No. CJF15014), the National Natural Science Funds of China (No. 71471123) and the Fundamental Research Funds for the Central Universities of China (No. skqy201621).

\section{References}

[1] J. Argyris, M. Haase, J. C. Heinrich, Finite element approximation to two-dimensional sine-Gordon solitons, Comput. Methods Appl. Mech. Engrg., 86 (1991), 1-26. 1

[2] Z. Asgari, S. M. Hosseini, Numerical solution of two-dimensional sine-Gordon and MBE models using Fourier spectral and high order explicit time stepping methods, Comput. Phys. Commun., 184 (2013), 565-572. 1, 2.4, 5.3

[3] A. G. Bratsos, A modified predictor-corrector scheme for the two-dimensional sine-Gordon equation, Numer. Algorithms, 43 (2006), 295-308. 1, 1, 3.2

[4] A. G. Bratsos, The solution of the two-dimensional sine-Gordon equation using the method of lines, J. Comput. Appl. Math., 206 (2007), 251-277. 1, 1, 3.2

[5] W. Chen, W. Feng, C. Wang, S. Wise, A second order energy stable scheme for the Cahn-Hilliard-Hele-Shaw equations, ArXiv, 2016 (2016), 34 pages. 2.1

[6] K.-L. Cheng, W.-Q. Feng, S. Gottlieb, C. Wang, A Fourier pseudospectral method for the "good" Boussinesq equation with second-order temporal accuracy, Numer. Methods Partial Differential Equations, 31 (2015), 202-224. 1

[7] R. J. Cheng, K. M. Liew, Analyzing two-dimensional sine-Gordon equation with the mesh-free reproducing kernel particle Ritz method, Comput. Methods Appl. Mech. Engrg., 245/246 (2012), 132-143. 1

[8] K.-L. Cheng, C. Wang, S. M. Wise, X.-Y. Yue, A second-order, weakly energy-stable pseudo-spectral scheme for the CahnHilliard equation and its solution by the homogeneous linear iteration method, J. Sci. Comput., 69 (2016), 1083-1114. 2.1

[9] M.-R. Cui, High order compact alternating direction implicit method for the generalized sine-Gordon equation, J. Comput. Appl. Math., 235 (2010), 837-849. 1, 3.2

[10] M. Dehghan, A. Ghesmati, Numerical simulation of two-dimensional sine-Gordon solitons via a local weak meshless technique based on the radial point interpolation method (RPIM), Comput. Phys. Comm., 181 (2010), 772-786. 1

[11] M. Dehghan, D. Mirzaei, The dual reciprocity boundary element method (DRBEM) for two-dimensional sine-Gordon equation, Comput. Methods Appl. Mech. Engrg., 197 (2008), 476-486. 1

[12] M. Dehghan, A. Shokri, A numerical method for solution of the two-dimensional sine-Gordon equation using the radial basis functions, Math. Comput. Simulation, 79 (2008), 700-715. 1

[13] J.-S. Hu, K.-L. Zheng, M.-B. Zheng, Numerical simulation and convergence analysis of a high-order conservative difference scheme for SRLW equation, Appl. Math. Model., 38 (2014), 5573-5581. 1

[14] R. Jiwari, S. Pandit, R. C. Mittal, Numerical simulation of two-dimensional sine-Gordon solitons by differential quadrature method, Comput. Phys. Commun., 183 (2012), 600-616. 1, 5.3

[15] S. Li, L. Vu-Quoc, Finite difference calculus invariant structure of a class of algorithms for the nonlinear Klein-Gordon equation, SIAM J. Numer. Anal., 32 (1995), 1839-1875. 1

[16] J. K. Perring, T. H. R. Skyrme, A model unified field equation, Nuclear Phys., 31 (1962), 550-555. 1

[17] W. Strauss, L. Vazquez, Numerical solution of a nonlinear Klein-Gordon equation, J. Comput. Phys., 28 (1978), $271-278$. $1,2.1$

[18] L.-D. Wang, W.-B. Chen, C. Wang, An energy-conserving second order numerical scheme for nonlinear hyperbolic equation with an exponential nonlinear term, J. Comput. Appl. Math., 280 (2015), 347-366. 1, 4.3

[19] C. Wang, S. M. Wise, An energy stable and convergent finite-difference scheme for the modified phase field crystal equation, SIAM J. Numer. Anal., 49 (2011), 945-969. 1 
[20] G. B. Whitham, Linear and nonlinear waves, Reprint of the 1974 original, Pure and Applied Mathematics (New York), A Wiley-Interscience Publication, John Wiley \& Sons, Inc., New York, (1999). 1

[21] J. X. Xin, Modeling light bullets with the two-dimensional sine-Gordon equation, Phys. D, 135 (2000), 345-368. 1

[22] X.-J. Yang, A new integral transform method for solving steady heat-transfer problem, Therm. Sci., 20 (2016), S639-S642. 1

[23] X.-J. Yang, A new integral transform with an application in heat-transfer problem, Therm. Sci., 20 (2016), S677-S681.

[24] X.-J. Yang, A new integral transform operator for solving the heat-diffusion problem, Appl. Math. Lett., 64 (2017), 193197. 1

[25] X.-J. Yang, F. Gao, H. M. Srivastava, Exact travelling wave solutions for the local fractional two-dimensional Burgers-type equations, Comput. Math. Appl., 73 (2017), 203-210. 1

[26] X.-J. Yang, J. A. Tenreiro Machado, D. Baleanu, C. Cattani, On exact traveling-wave solutions for local fractional Korteweg-de Vries equation, Chaos, 26 (2016), 5 pages. 1

[27] J. Zagrodziński, Particular solutions of the sine-Gordon equation in $2+1$ dimensions, Phys. Lett. A, 72 (1979), $284-286$. 1

[28] K.-L. Zheng, J.-S. Hu, High-order conservative Crank-Nicolson scheme for regularized long wave equation, Adv. Difference Equ., 2013 (2013), 12 pages. 1 